\title{
BENCHMARKING IMAGE COMPRESSION: A REVIEW
}

\author{
Niveditta Thakur ${ }^{1}$, Aayush Gupta ${ }^{2} \&$ Sunidhi Raju ${ }^{3}$
}

Abstract- Images are considered as the major information bundles in the world, they can convey a lot of additional info to the receptor then some pages of written information. Due to this very reason, constriction become a significant field of analysis nowadays. Despite the advancement in the Technology in storage and transmission, the demand on the capacity limits and transfer speed of communication surpass the availability. Hence, compression has signified be a valuable technique. The cornerstone of compression is to scale back interrelationship and overabundance. Compression can be studied on the basis of two parameters i.e. lossless or lossy, lossless compression transforms the precise original image from the constrict ed image whereas lossy compression cannot re-establish the initial image from the constricted data. Many constriction algorithms are available to compress data of any format. This paper includes the examination and correlation of a specific set of lossless and lossy constriction algorithm.

Keywords - Deflate; Google WebP; Graphical Interchange Format; Lossless Compression; Lossy Compression; Normalized Cross-Correlation(NK).

\section{INTRODUCTION}

Compression turned out to be an essential part of today's digital world. Compression not only minimises bandwidth and storage requirements but also reduces the time required to transmit information. Consequently, the methods of constricting data prior to storage or transmission are of significant practical and commercial interest Most compression schemes are lossy, where high constriction ratios are obtained by sacrificing info within certain allowable degradation limits. Documents preserving, medical and satellite imaging or any application demanding ultra-high image fidelity etc. are the important areas, [1-2] that require lossless compression (i.e., reconstruct the constricted data without any loss of information). The performance of an image compression algorithm can be specified in the term of quality Benchmarking Metrics.

Constriction system mainly comprises of two blocks i.e. Encoder and Decoder. Input is fed into encoder which encodes the data to make it suitable for transmission and Decoder receives compressed data and reconstructs the output. If the system is error free, then the output is exact replica of input. Encoder and decoder comprise of three and two blocks respectively. Encoder consists of Mapper, Quantizer and Symbol Encoder. The mapper converts the image into a format arranged to reduce spatial domain redundancies [3] in the input image. Quantizer is generally a lossy process which degrades mapper's output according to some pre-established criterion. Symbol Encoder generates a variable-length code [4] to present quantizer's output and drafts the result according to the coding method used. Decoder consists of inverse Mapper and symbol Decoder.

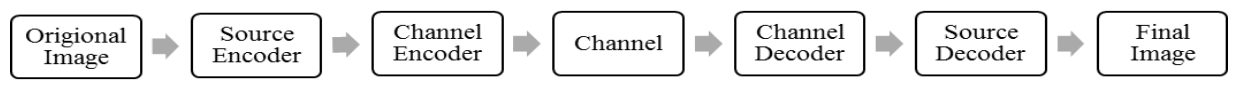

Figure 1. Image Compression/Decompression Model

This paper is arranged in the way as Section I presents Introduction, Section II overviews Compression Procedures Section III specifies Lossless Approaches, Section IV convey Lossy Approaches, Section V describes the Comparative analysis of Compression Measures Section VI presents Concluding remarks and Future Scope, and at last References.

\section{COMPRESSION PROCEDURES}

In lossless constriction procedures, the resultant image, after constriction, is indistinguishable to the initial image. However lossless constriction can accomplish only an unobtrusive amount of constriction whereas, lossy constriction is capable of achieving higher compression with degraded quality.

\footnotetext{
${ }^{1}$ Faculty University Institute of Information Technology, Himachal Pradesh University, Shimla-171005, Himachal Pradesh, India

${ }^{2}$ Student University Institute of Information Technology, Himachal Pradesh University, Shimla-171005, Himachal Pradesh, India

${ }^{3}$ Faculty University Institute of Information Technology, Himachal Pradesh University, Shimla-171005, Himachal Pradesh, India
} 


\subsection{Lossless Image Compression-}

The principle behind lossless image compression is to assign every bit of image with the shortest possible code without loss of any data, resulting in speeding up transmission and reducing storage space. This reproduces the initial image nearly without any quality loss. This is a reversible constriction algorithm.

Some of the Lossless Constriction Algorithms are:

- Walsh-Hadamard Transform

- Principal Component Analysis

- Deflate(PNG)

- DWT

- WebP

\subsection{Lossy Image Compression-}

The principle behind lossy technique is to remove interpixel redundancy [5] as a result, higher constriction rate can be achieved but a considerable loss in the quality. Most lossy compression algorithms suffer from generation loss [6] i.e. repeatedly constriction and deconstruction which causes progressive loss in quality. This is an irreversible constriction algorithm.

Some of the Lossy Constriction Techniques are:

- DCT

- GIF

- JPEG

\subsection{Compression Analysis Parameters -}

The performance of various constriction Techniques can be specified in terms of Average Difference(AD), Compression efficiency [7-8], Laplacian Mean Squared Error(LMSE), Normalized Cross-Correlation(NK), Mean Squared Error(MSE), Maximum Difference (MD), Universal Image Quality Index (UIQI), Peak Signal to Noise Ratio (PSNR), Mean Absolute Error (MAE), Structural Content (SC), Structural Similarity Index Metric (SSIM) and complexity of Algorithm. Compression ratio is the ratio of the size of the initial image to the size of the final image and the bit rate is the number of bit per pixel required by the final image. The aggregation of these two is called compression Efficiency. Let CR be the constriction ratio, and BR be the bit rate and $b$ be the number of bits per pixel of the initial image, the following equation is readily obtained.

$\mathrm{CR}=\mathrm{b} / \mathrm{BR}$

The efficiency of the constriction method can be correlated to the entropy [9] of the original image. The source entropy is described as the amount of information accommodated in the source. Suppose that the pixel values range from 0 to Y-1. Let bi be the probability of value i. So, the Entropy $(\mathrm{H})$ will be calculated as:

$\mathrm{H}(\mathrm{I})=-\sum_{\mathrm{i}=0}^{\mathrm{Y}-1} \mathrm{~b}_{\mathrm{i}} \log \mathrm{b}_{\mathrm{i}}$

Entropy is measured in bits per pixel. The efficiency of a constriction method is calculated by determining how closely its bitrate approximate the source entropy. The complexity of a compression algorithm is calculated by the number of crucial steps performed by the algorithm. This is an important factor for applications where speed is crucial. A resemblance of image with the initial image is sufficient for most purposes, as long as the error between initial and the final image is tolerable. Major error metrics used to study image compression are MSE [10] and PSNR. Cumulative squared error between the constricted and the test image is called MSE, whereas the calculation of the peak error is called PSNR [11]. The mathematical formulae for the two are:

$\operatorname{MSE}=\frac{1}{\mathrm{XY}} \sum_{\mathrm{y}=1}^{\mathrm{Y}} \sum_{\mathrm{x}=1}^{\mathrm{X}}[\mathrm{I}(\mathrm{x}, \mathrm{y})-\mathrm{J}(\mathrm{x}, \mathrm{y})]^{2}$

PSNR $=20 \times \log _{10}(255 / \sqrt{\text { MSE }})$

Here $I(x, y)$ is the initial image, $J(x, y)$ is the Resultant image and $W, X$ are the dimensions of the images. A lower value for MSE and a higher value of PSNR tends to good result.

$\mathrm{AD}[12]$ is simply the average of difference between the initial and final image. Larger the value of AD poorer the quality of image It is given by the equality.

$\mathrm{AD}=1 / \mathrm{XY} \sum_{\mathrm{x}=1}^{\mathrm{X}} \sum_{\mathrm{y}=1}^{\mathrm{Y}}(\mathrm{I}(\mathrm{x}, \mathrm{y})-\mathrm{J}(\mathrm{x}, \mathrm{y}))$

$\mathrm{MD}$ [13] is the maximum difference between the original and resultant image. Larger the value worst the quality. It is given by the equation.

$M D=\max |I(x, y)-J(x, y)|$

MAE [14] is the average of absolute difference between the source image and final image. Large value of MAE leads to poor quality. It is given by the equation.

$M A E=1 /{ }_{X Y} \sum_{x=1}^{X} \sum_{y=1}^{Y}\lfloor I(x, y)-j(x, y)\rfloor$

NK [15] measures the similarity between two images and is given by equality.

$N K=\frac{\sum_{x=1}^{X} \sum_{y=1}^{Y}(I(x, y) \times J(x, y))}{\sum_{x=1}^{X} \sum_{y=1}^{Y}(I(x, y))^{2}}$ 
SC [16] is also a correlation based measure and measures similarity between two objects. Structural Content (SC) is given by the equality.

$S C=\frac{\sum_{x=1}^{X} \sum_{y=1}^{Y}(J(x, y))^{2}}{\sum_{x=1}^{X} \sum_{y=1}^{Y}(I(x, y))^{2}}$

LMSE [17] is based upon edges measurement. The large value of LMSE depict that image is poor quality. LMSE is described by given mathematical formula:

$L M S E=\frac{\sum_{x=1}^{X} \sum_{y=1}^{Y}[P(I(x, y))-P(J(x, y))]^{2}}{\sum_{x=1}^{X} \sum_{y=1}^{Y}[P(w(x, y))]^{2}}$

where $\mathrm{P}(\mathrm{x}, \mathrm{y})$ is Laplacian operator:

$\mathrm{P}(\mathrm{x}(\mathrm{m}, \mathrm{n}))=\mathrm{x}(\mathrm{m}, \mathrm{n})+\mathrm{x}(\mathrm{m}, \mathrm{n})+\mathrm{x}(\mathrm{m}, \mathrm{n})+\mathrm{x}(\mathrm{m}, \mathrm{n})-4 \mathrm{x}(\mathrm{m}, \mathrm{n})$

The SSIM [18] index is the quality measure in which one images are being compared on the basis of similarity, provided the original image is of perfect quality.

$\operatorname{MSSIM}(I, J)=1 / X_{x=1}^{\sum_{x}}\left[I\left(i_{x}, j_{X}\right)\right]\left[c\left(i_{x}, j_{X}\right)\right]\left[e\left(i_{x}, j_{X}\right)\right]$

UIQI [19] models distortion in the image via combination of three factors: luminance distortion, loss of correlation, and contrast distortion. Equation for UIQI is:

$\mathrm{Q}=\frac{\sigma_{\mathrm{xy}}}{\sigma_{\mathrm{x}} \sigma_{\mathrm{y}}} \cdot \frac{2 \mathrm{xy}}{\mathrm{x}^{2}+\mathrm{y}^{2}} \cdot \frac{2 \sigma_{\mathrm{x}} \sigma_{\mathrm{y}}}{\sigma_{\mathrm{x}}{ }^{2}+\sigma_{\mathrm{y}}{ }^{2}}$

\section{LOSSLESS APPROACHES}

Lossless constriction is a part of algorithms that allows the initial data to be fully reconstructed from the resultant data. Lossy compression however allows reconstruction upto an approximation of the original data, However, it usually improves compression rates. Lossless constriction is used in cases where slight alteration of the initial data could be destructive.

\subsection{Walsh Hadamard Transform-}

With the use of WHT [20], quantization and encoding an image can be constricted. In this method, RGB components are converted to YCbCr. The original image is divided into $8 \times 8$ blocks of pixels. WHT optimises the processing time of image Constriction. Each block is calculated by using the WHT. Then probability is calculated by image quantization and after applying quantization, Huffman code is calculated for each unique symbol so as to compress image using Huffman coding. In WHT objective is to decrease the bandwidth requirement for the transmission of images.

\subsection{Principal Component Analysis-}

PCA[21] also known as K-L expansion, that is one of the classical dimensionality reduction method used for feature extraction which has been widely used in variety of areas such as signal processing, pattern recognition and data mining. Application of PCA in the area of image constriction can be seen in various forms, i.e. as a a pre-processing or post-processing step in combination with other techniques or a standalone image constriction technique,. To compress an image, firstly, mean of each row is found and subtracted from the corresponding row. The mean subtracted is the average across each dimension. Resulting matrix is called adjusted matrix A. Then its covariance matrix is found and its eigenvalues and eigenvectors are calculated. These are rather important, as they provide us with information about the patterns in the data. Next, the eigenvectors are sorted according to their significance and a matrix F composed of eigenvectors is formed, where each vector is a column. Number of eigenvectors are discarded starting from the least important one. The number of vectors $(\mathrm{k})$ kept depends on the loss of information can be afforded, regarding the particular application. To obtain compressed data set, transpose of the eigenvector matrix is multiplied it by adjusted matrix:

$\mathrm{A}_{\mathrm{k}}=\mathrm{F}_{\mathrm{k}}^{\mathrm{T}} \times \mathrm{A}$

Decompression Procedure

$A=\left(F_{k}^{T}\right)^{-1} \times A_{k}$

If $\mathrm{k}=\mathrm{N}($ no loss), then

$\mathrm{A}=\mathrm{F}_{\mathrm{k}} \times \mathrm{A}_{\mathrm{k}}$

$A_{\text {origional }}=F_{k} \times A_{k}+M$

else

$\mathrm{A}^{\prime}=\mathrm{F}_{\mathrm{k}} \times \mathrm{A}_{\mathrm{k}}$

$A_{\text {origional }}^{\prime}=F_{k} \times A_{k}+M$

Here $M$ is a matrix of the mean values (averages) of the input dimensions. Hence, $A_{\text {origional }}$, compressed set $A_{k}$ and decompressed set $\mathrm{A}_{\text {origional }}^{\prime}$ where $\mathrm{A}_{\text {origional }}^{\prime}=\mathrm{A}_{\text {origional }}$ if $\mathrm{k}=\mathrm{N} . \mathrm{F}_{\mathrm{k}}, \mathrm{A}_{\mathrm{k}}$ and $\mathrm{M}$ are the only values that are needed to be stored, since they are sufficient in obtaining Result. 


\subsection{Deflate-}

Deflate [22] is a lossless data constriction algorithm. A combination of the LZ77 algorithm and Huffman Coding is used in this algorithm. The data is constricted as a agggregation of matching strings and encoded bytes, here strings are to be found in the preceding uncompressed data. Each match is a length and a distance back from the current position. The literals and lengths are combined into a single Huffman code, and the distances in another Huffman code. Longer lengths and distances fall into bins, followed by extra bits to determine which entry in the bin to use. The stream comprises of a series of length codes, where it is succeeded by the distance code. A distance may be less than the length, in that case the previous available data is copied, and then copied again until the length is reached. The lengths can be in $3 \ldots . .258$, and the distances can be in $1 . .32768$, where 32768 bytes is the amount of previous data retained. This approach to code a sequence of literals and matches is called "LZ77".

The deflate stream is broken into blocks, where each block starts with the definition of the Huffman codes for that block, followed by the literal/length and distance codes, and finally an end-of-block code. The description of the Huffman codes consists of the code lengths of each symbol, where description is itself Huffman and run-length encoded. The last block is marked as such, so the deflate format is self-terminating. There are also fixed Huffman code blocks, which use a single predefined literal and distance code, and stored blocks which simply copy the data uncompressed..

The deflate algorithm searches for matching strings in the preceding data. It is in this search that most of the effectiveness of the compressor is determined, as well as its speed. In zlib a hash table is constructed on all preceding three-byte sequences (clearing old ones as the window slides out). Hash hits result in directly looking for a matching string at the associated distance back. User-selected compression level determines when to stop looking for better matches, completeness of the hash table and consequently the speed. Other deflate compressors use more complete, but slower approaches such as suffix trees to find previous matches. The deflate effectiveness can also be optimized by smartly choosing when to start a new block, which allows the algorithm to adapt to changing statistics in the data.One of the Major Application of Deflate Algorithm is PNG. PNG is an acronym for Portable Network Graphics. PNG file format is used for storing, and displaying high quality images. PNG supports lossless constriction, opacity configuration, and a range of color depths. Gamma correction, standard color space for precise reproduction of image colors on a wide range of systems and embedded textual info are the advance features supported by PNG.

\subsection{Discrete Wavelet Trsnsform-}

Waveform with an average value of zero is called a wavelet. In general ideal properties for compact signal representation and orthogonality are displayed by the wavelets. A signal can be devided into many scaled representations of the mother wavelet. This can be used to devide a signal into its child wavelets. Once this is done the coefficients of the wavelets can be destroyed to remove useless details. Small wavelets are used to confine important and very fine and details in a signal, while very large wavelets are used to identify insignificant details. Wavelets can be choosen from various different wavelets i.e. Morlet, Daubechies, etc. More sparce representation of signals can be produced using different wavelets. To attain image compression many diferrent kinds of wavelets must be examined and best among them must be choosen.

A wavelet function $\Psi(\mathrm{x})$ has two main properties,

$\int_{-\infty}^{0} \psi(\mathrm{x}) \mathrm{dx}=0$

That is, the function has wavy appearance.

$\int_{-\infty}^{0}|\psi(x)|^{2} d y<\infty$

That is, the most of the energy in $\Psi(\mathrm{t})$ is confined to a finite duration.

Sub-bands are created by decomposition of image into coefficients and then these coefficients are compared woth the threshold. Coefficients below threshhold are set to zero and those above are encoded with the lossless constriction technique.

The steps of DWT Compression Algorithm are described below:

- Decompose: Wavelet and level W are chosen. Wavelet is computed and signals are decomposed at level W.

- Threshold detail coefficients: For each level a threshold is selected i.e. from 1 to $\mathrm{W}$ and then to the resultant coefficients, hard thresholding is applied

- Reconstruct: Wavelet reconstruction is computed using the initial approximation concomitants of level $\mathrm{W}$ and modified detail concomitants of levels from 1 to $\mathrm{W}$.

\section{$3.5 \mathrm{WebP}-$}

Google developed a image format named WebP [24] that can optimize image for faster transmission on the Web. In WebP image is first transferred using various techniques and then entropy encoding is done on the transformed data. For transformations various methods like Spatial prediction of pixels, colour transform using local emerging palettes, alpha replacement and packing multiple pixels into one. Variant of LZ77 [25] and Huffman coding[26] are used for entropy encoding, which uses compact sparse values. 
- Predictor (Spatial) Transform: Since neighbouring pixels are always correlated so spatial prediction is used to reduce entropy. The image is divided into multiple square regions and all the pixels in that region use the same prediction mode.

- Colour (de-correlation) Transform: In this step the R, G and B values of each pixel are decorrelated. The green $(\mathrm{G})$ values are kept as it is and red (R) and Blue(B) are transformed on the basis of green and then based on red.

- Subtract Green Transform: In this step, green values are subtracted from each red and blue pixel.

- Colour cache coding: New pixels are reconstructed by the use of already seen image fragments. If no interesting match is found, then local palette is used and palette is updated continuously.

- LZ77 Backward Reference: Tuples of length and distance code is called Backward References. Length and distance values are stored by the application of LZ77 prefix coding. In LZ77 prefix coding large values are divided into two parts: the prefix code and the extra bits. The prefix code is stored using an entropy code, while the extra bits are stored as they are.

The format uses sub-resolution pictures, recursively embedded into the format itself, for storing statistical information regarding the images, such as the used entropy codes, spatial predictors, color space conversion, and color table. LZ77, Huffman coding, and a color cache are used for compression of the bulk information.

\section{LOSSY APPROACHES}

Lossy constriction or irreversible constriction is the class of data encoding methods that uses partial data discarding and inexact approximations to represent data. These techniques are used to reduce info size for storage, handling, and transmitting content. Well-designed lossy compression technology significantly reduces file sizes before deviation from the initial image is noticed by the end-user. Most common use of this compression is to compress digital data, especially in applications such as streaming internet telephony and media.

\subsection{Discrete Wavelet Trsnsform-}

In DCT [27] signal is transformed from spatial representation to frequency representation. Sum of sinusoids of varying frequencies and magnitudes is represented by DCT. DCT coefficients are normalized after the computation according to a quantization table with different scales provided by the JPEG standard computed by psycho-visual evidence. Selection of quantization table affects the compression ratio and entropy. The value of quantization is inversely proportional to quality of final image. In a lossy constriction technique, during Quantization step less important frequencies are destroyed, and then remaining frequencies are used to retrieve the image in decomposition process. Firstly, image is loaded then RGB to GRAY conversion is done, then it is divided into small WXW blocks then working from top to bottom and left to right and DCT is applied to each block. Each block 's elements are constricted by Quantization forming a quantization matrix called $\mathrm{Q}_{\text {matrix }}$ and approximating to the nearest integer value. This $\mathrm{Q}_{\text {matrix }}$ is decided by the user keeping in mind that Quality levels ranging from 1 to 100 are given by it, where 1 gives the highest CR and poor image Quality while 100 gives lowest CR and best Quality of decompressed image.

Now data is encoded and transmitted to the receiver in the form of 1D array. This transmitted sequence saves in the text format. To re-constrict the image, quantized DCT coefficients are decoded by the user and inverse 2D DCT of each block is computed, then reconstructed blocks are put together into a single image. The dequantization is achieved by multiplying each element of the received data by corresponding element in the quantization matrix $\mathrm{Q}_{\text {matrix }}$, then 128 added to each element for getting level shift. These blocks are merged and arranged in same order in which they were decomposed for compression to get the de-constricted image.

\subsection{Graphics Interchange Format-}

This invention relates to creating a compressed electronic image using a dictionary based variable lossy compression algorithm. A dictionary based compression algorithm works by storing a table of all of the unique strings that occur in an image together. A common dictionary based constriction algorithm is the Lempel-Ziv-Welch [28] (LZW) algorithm. The LZW algorithm is used to compress and store images in the Graphical Interface Format [29] (GIF), and Works by representing two different byte strings in a file undergoing constriction by the same string identifier in the dictionary or compression table of the corresponding compressed file. The modified LZW algorithm operates by comparing byte strings that are stored in the file's compression table to the byte string in a file undergoing compression, and determining whether the two byte strings are sufficiently different to Warrant storing the byte string together with a new string identifier in the compression table. If the difference between the byte string of image and the byte string already stored in the compression table is little then it is represented by the same string identifier representing the existing compression table byte string. As a result, the information content of byte strings that are slightly different from the previously stored compression table byte strings is lost in the compressed file.

\subsection{Joint Photographic Expert Group-}

JPEG [30] uses lossy form of constriction based on the DCT. This mathematical operation converts pixels from the 2D domain to the frequency domain. Sharp transitions in intensity, and colour hue are discarded based on perspective model of 
human phycho visual intensity. This process of reducing info in the frequency domain is called quantisation. Now quantised coefficients are sequenced and packed into output stream. User has full control over JPEG implemetation. User can control various parameters like quality, compression ratio exhibiting the trade off between file size and quality. This method is generally lossy i.e. affecting the image quality. Additionally there are lossless JPEG constriction standards. However this mode is not generally supported by products

\section{LOSSY APPROACHES}

Image Compression can be lossy or lossless. Compression algorithm works differently on different environments or images, so 5 different types of images are taken as follows: IM_01 represents Fingerprint image, IM_02 represents Medical Image, IM_03 and IM_04 represents General Test Image and finally IM_05 presents Saturn rings that's an Astral image.

Table 1 describes the Performance Evaluation of various compression schemes on the basis of various quality measures like MSE, PSNR, MAE, AD, SSIM, UIDI, Compression Ratio and Compression Percentage on different images. As compression algorithms are based on redundancy so every algorithm behaves in variant ways on different images showing unlike results. Let's Consider IM_01 for result analysis. IM_01 is compressed by 4 compression algorithms and their quality measures and compression rate are studied.

Table -1 Performance Evaluation of Various Compression Schemes

\begin{tabular}{|c|c|c|c|c|c|c|c|c|c|}
\hline \multirow{2}{*}{ Images } & \multirow{2}{*}{$\begin{array}{l}\text { Compression } \\
\text { Technique }\end{array}$} & \multicolumn{6}{|c|}{ Quality Measures } & \multirow{2}{*}{$\begin{array}{l}\text { Compression } \\
\text { Ratio }\end{array}$} & \multirow{2}{*}{$\begin{array}{l}\text { Compression } \\
\text { Percentage }\end{array}$} \\
\hline & & MSE & PSNR & MAE & $\mathrm{AD}$ & SSIM & UIDI & & \\
\hline \multirow{4}{*}{ IM_01 } & JPEG & 5.7645 & 20.2616 & 1.8471 & 4.9113 & 0.8035 & 0.93373 & 0.1586 & $85 \%$ \\
\hline & JPEG2000 & 7.2116 & 19.7752 & 2.0909 & 4.9047 & 0.7717 & 0.91088 & 0.3112 & $69 \%$ \\
\hline & GIF & 191.03 & 2.6599 & 130.295 & 130.28 & 0.0055 & 0.01039 & 0.8877 & $12 \%$ \\
\hline & PNG & 0 & Infinite & 0 & 0 & 1 & 1 & 0.8075 & $20 \%$ \\
\hline \multirow{4}{*}{ IM_02 } & $\overline{J P E G}$ & 6.3509 & 20.0513 & 1.6809 & 0.1814 & 0.8023 & 0.91076 & 0.1267 & $98 \%$ \\
\hline & JPEG2000 & 7.3632 & 19.7301 & 1.8408 & 0.1690 & 0.7407 & 0.84785 & 0.2675 & $74 \%$ \\
\hline & GIF & 136.8 & 3.3900 & 93.8296 & 54.5155 & 0.0040 & 0.00540 & 0.6259 & $38 \%$ \\
\hline & PNG & 0 & Infinite & 0 & 0 & 1 & 1 & 0.6289 & $38 \%$ \\
\hline \multirow{4}{*}{ IM_03 } & JPEG & 3.4942 & 21.3487 & 1.2939 & 1.2870 & 0.7148 & 0.83809 & 0.1256 & $88 \%$ \\
\hline & JPEG2000 & 7.8053 & 19.6035 & 2.0973 & 1.2830 & 0.4692 & 0.60646 & 0.1681 & $84 \%$ \\
\hline & GIF & 80.401 & 4.5391 & 71.881 & 20.1725 & 0.0012 & 0.00331 & 0.6764 & $33 \%$ \\
\hline & PNG & 0 & Infinite & 0 & 0 & 1 & 1 & 0.6181 & $39 \%$ \\
\hline \multirow{4}{*}{ IM_04 } & JPEG & 14.8855 & 18.2016 & 2.9072 & 0.1200 & 0.7208 & 0.90274 & 0.1938 & $81 \%$ \\
\hline & JPEG2000 & 7.0489 & 19.8248 & 2.0858 & 0.0930 & 0.7882 & 0.92441 & 0.6499 & $36 \%$ \\
\hline & GIF & 65.829 & 4.9733 & 64.8815 & 27.4865 & 0.0199 & 0.01376 & 0.9367 & $7 \%$ \\
\hline & PNG & 0 & Infinite & 0 & 0 & 1 & 1 & 0.8418 & $16 \%$ \\
\hline \multirow{4}{*}{ IM_05 } & JPEG & 4.6809 & 20.7137 & 1.5445 & 1.0700 & 0.4653 & 0.57148 & 0.0837 & $92 \%$ \\
\hline & JPEG2000 & 6.0016 & 20.1741 & 1.8494 & 1.0731 & 0.4223 & 0.51544 & 0.0766 & $93 \%$ \\
\hline & GIF & 91.103 & 4.2677 & 76.3086 & 14.5638 & 0.0076 & 0.00844 & 0.7813 & $22 \%$ \\
\hline & PNG & 0 & Infinite & 0 & 0 & 1 & 1 & 0.7229 & $28 \%$ \\
\hline
\end{tabular}

Similarly, other four images are also studied in the same pattern. While comparing all compression techniques only GIF is one such technique which is unable to maintain the quality and compression standards up to a level; a level other techniques have maintained so far. In case of PNG the result is obvious as it's a lossless compression algorithm so it will yield high quality whereas its compression efficiency is very less as compared to other algorithms i.e. JPEG and JPEG2000. JPEG and JPEG2000 yielded nearly similar results in all the cases however there are variations in case of IM_04. The simple reason for this out of symmetry result is the variation in the image since JPEG [30] is based on inter-pixel redundancy [5] and IM_04 has lot more variations then a normal picture. 


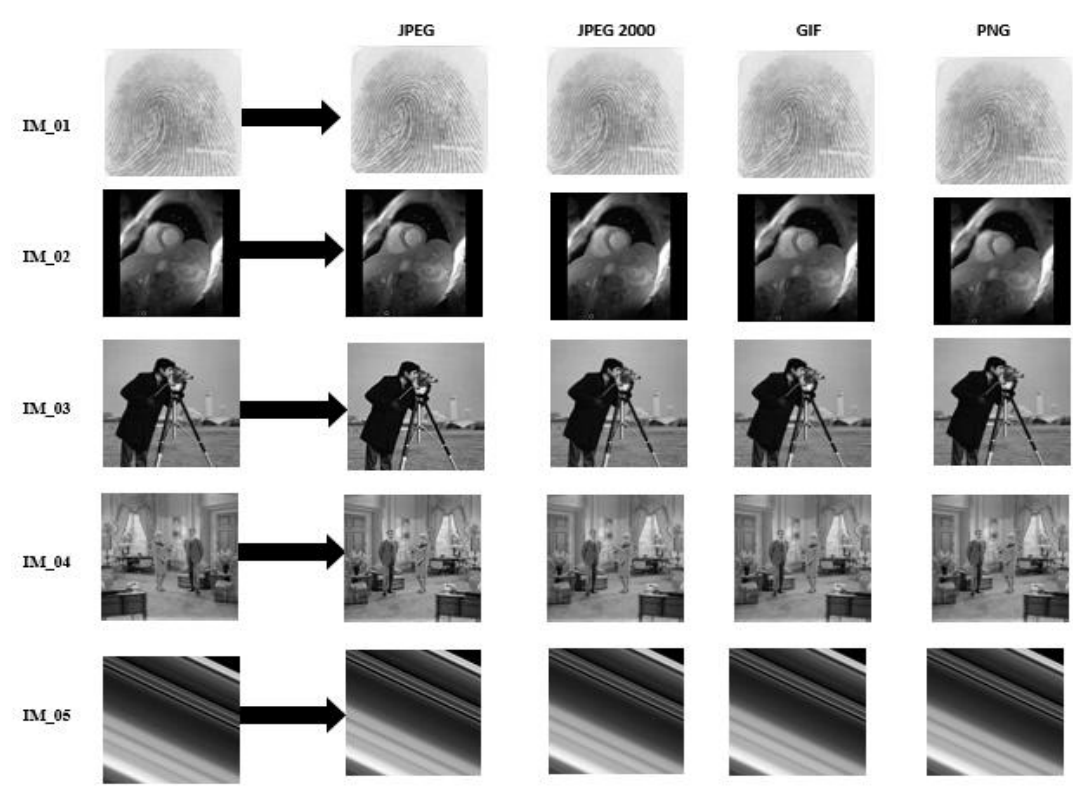

Figure 2. Application of various Compression Techniques "JPEG", "JPEG2000", "GIF" and "PNG" on test images "IM 01" "IM 05"

\section{CONCLUDING REMARKS AND FUTURE SCOPE}

Image compression is very important for efficient transmission and storage of images. The work in this paper primarily focuses at comparing the most widely used techniques in the image compression domain on various images. The techniques are successfully studied and implemented on various images. The compression algorithms are implemented using the MATLAB 2016a software on different test images. According to the result calculated in table 1 JPEG gave higher compression but quality was not maintained up to the mark whereas PNG has maintained high quality but gave low compression rate. Every compression is best in its own dimensions. In the near future, more compression Techniques will be considered which can compress with higher compression ratio and maintain the quality of image. In future, many technologies can arise in which compression can be done not only on the basis of redundancy but on symmetry in the images too.

\section{REFERENCES}

[1] Menzel and Ralph E, inventor; Bennethum Computer Systems, assignee. "Method for preserving data in an electronic document," United States patent US 5,937,416. 1999 Aug 10.

[2] A. Silverstein and J. E. Farrell, "The relationship between image fidelity and image quality", Proceedings of IEEE International Conference Image Processing, pp. 881-884, 1996.

[3] Trieu-Kien Truong, Jyh-Horng Jeng and Irving S. Reed, "A fast encoding algorithm for fractal image compression using the DCT inner product," IEEE Transaction Image Processing, vol. 9, Issue no. 4, pp. 529-535, 2000.

[4] S. Amritpal, "Enhanced approach of Entropy Coding in Image Compression." PhD diss., THAPAR UNIVERSITY PATIALA, 2013

[5] Yang, Ming, and Nikolaos Bourbakis, "An overview of lossless digital image compression techniques," In Circuits and systems, 2005. 48th Midwest symposium on, pp. 1099-1102, 2005.

[6] Ukrit, M. Ferni, A. Umamageswari, and G. R. Suresh. "A survey on lossless compression for medical images." International Journal of Computer Applications, vol. 31, issue no. 8, pp. 47-50, 2011.

[7] Saffor Amhamed, Abdul Rahman Ramli, and Kwan-Hoong Ng, "A Comparative study of image compression between JPEG and wavelet," Malaysian Journal of computer science, vol. 14, issue no. 1, pp. 39-45, 2001.

[8] Sahni, S., B. C. Vemuri, F. Chen, C. Kapoor, C. Leonard, and J. Fitzsimmons. "Lossless Image Compression," IEEE Geoscience and Remote Sensing Symposium, pp. 1025-2048, 2003.

[9] Hassaballah, Makky, M. M. Makky, and Youssef B. Mahdy, "A fast fractal image compression method based entropy," Electronic Letters on Computer Vision and Image Analysis, vol. 5, issue no. 1, pp. 30-40, 2005.

[10] Gu, Ke, Shiqi Wang, Guangtao Zhai, Siwei Ma, Xiaokang Yang, and Wenjun Zhang, "Content-weighted mean-squared error for quality assessment of compressed images." Signal, Image and Video Processing, vol. 10, issue no. 5, pp. 803-810, 2016.

[11] K. Pooja and Yuvraj Sharma, "Comparison of different image enhancement techniques based upon PSNR \& MSE," International Journal of Applied Engineering Research, vol. 7, issue no. 11, pp. 2010-2014, 2012.

[12] Galbally, Javier, Sébastien Marcel, and Julian Fierrez. "Image quality assessment for fake biometric detection: Application to iris, fingerprint, and face recognition." IEEE transactions on image processing, vol. 23, isuue no. 2, pp. 710-724, 2014.

[13] C. Sasi varnan, A.Jagan, Jaspreet Kaur, Divya Jyoti, and Dr.D.S.Rao, "Image quality assessment techniques in spatial domain,” International Journal Computer Science and Telecommunication, vol. 2, issue no. 3, pp. 117-125, 2011.

[14] Eskicioglu, Ahmet M., Paul S. Frtsher, and Siyuan Chen, "Image Quality Measures and their performance," IEEE Transactions on Communication, vol. 43, issue no. 12, pp. 2959-2965, 1995. 
[15] Rao, Y. Raghavender, Nikhil Prathapani, and E. Nagabhooshanam, "Application of normalized cross correlation to image registra tion," International Journal of Research in Engineering and Technology, vol. 3, issue no. 05, pp. 12-16, 2014.

[16] Mayuresh Gulame, K.R. Joshi, R.S. Kamthe, "A Full Reference Based Image Quality Assessment", International Journal of Advanced Electrical and Electronics Engineering, vol. 2, issue no. 6, pp. 2278-8948, 2013.

[17] Vora, V. S., A. C. Suthar, Y. N. Makwana, and S. J. Davda. "Analysis of Compressed Image Quality Assessments," International Journal of Advanced Engineering \& Application, pp. 225-229, 2010.

[18] Wang, Zhou, Alan C. Bovik, Hamid R. Sheikh, and Eero P. Simoncelli, "Image quality assessment: from error visibility to structural similarity," IEEE transactions on image processing, vol. 13, isue no. 4, pp. 600-612, 2004.

[19] Zhou Wang and A.C. Bovic, “A universal Image Quality Index,” IEEE Signal Processing Letters, vol. 9, issue no. 3, pp. 1-4, 2002.

[20] Khin Thinda Win, Nang Aye Aye, "Image Compression based on modified Walsh-Hadamard Transform," 3rd International Society for Engineering Research and Development International Conference, ISBN: 978-93-85465-22-2, pp. 58-62, 2015.

[21] Stolevski, Stefan, "Hybrid PCA algorithm for image compression," 18th Telecommunication Forum TELFOR, pp. 685-687, 2010.

[22] Clauson, Jon D. "Variable lossy compression." U.S. Patent 7,062,088, issued June 13, 2006.

[23] M. Mozammel Hoque Chowdhury and Amina Khatun "Image Compression Using Discrete Wavelet Transform" International Journal of Computer Science, vol. 9, issue no. 4, ISSN (Online): 1694-0814, pp. 327-330, 2012.

[24] "Compression Techniques | Webp | Google Developers". Google Developers. N.p., 2017. Web. 26 Mar. 2017.

[25] Ziv, J., and A. Lempel, "A Universal Algorithm for Sequential Data Compression," IEEE Transactions on Information Theory, vol. IT-23, issue no. 3, pp. 337-343, 1977.

[26] Nehal Markandeya and Sonali Patil, "Image Compression using Huffman Coding," International Journal of Engineering and Computer Science, vol. 6, issue no. 1, ISSN: 2319-7242, pp. 19999-20002, 2017.

[27] Maneesha Gupta and Dr. Amit Kumar Garg, "Analysis of Image Compression Algorithm using DCT," International Journal of Engineering Research and Applications, vol. 2, issue no. 1, ISSN: 2248-9622, pp.515-521, 2012.

[28] Dheemanth H N, "LZW Data Compression," American Journal of Engineering Research, vol. 03, issue no. 02, ISSN: 2320-0847, pp. $22-26,2014$.

[29] Ali, H.A. and Ne'ma, B.M., "Effective variations on opened gif format images," International Journal of Computer Science and Network Security, vol. 8 , isuue no. 5, pp. 70-75, 2008.

[30] Alain M. Leger, Takao Omachi and Gregory K. Wallace, “JPEG still picture compression algorithm,” Optical Engineering, vol. 30, issue no. 7, pp.947-954, 1991. 